\title{
Attendance rate in an alcohol problem clinic
}

\author{
Imad M. Ali and Andrew J. McBride
}

\begin{abstract}
The high rate of non-attendance for first appointments at poychiatilic clinics is wasteful of hecith senvice resources. Previous studies have demonstrated reductions in the non-attendance rates when referred patients were asked to contact the clinic to conifim that they wished to be seen before an appointment was sent. The effect of introducing such a process to a community based alcohol service was investigated. Results showed that although there was a reduction in the non-aftendance rate, the overall effect was to cliscourage patients from aftending the clinic, resulting in a large reduction in the proportion of all referrats seen. Altemative approaches are discussed.
\end{abstract}

Non-attendance of new out-patients at psychiatric clinics is a common problem which is wasteful of clinicians' time and other resources. The rate of non-attendance for first appointments has been shown to be around $30 \%$ in a general psychiatric clinic (Skuse, 1975), between 15-50\% at child psychiatry clinics (Plunkett. 1984; Gould et al, 1985; Cottrell et ah, 1988) and between $12-52 \%$ in alcohol treatment clinics (Addenbrooke \& Rathod, 1990; Goldbeck, 1993).

One study in a general adult psychiatric clinic showed that patients were more likely to attend if they had a previous psychiatric history, the appointment was held at a health clinic rather than in a psychiatric hospital, and patients received a personal letter rather than an impersonal, standard appointment card (Hillis \& Alexander, 1990). In child psychiatry clinics the attendance rate was not increased by sending families a hand-written appointment letter (Davison, 1992) but was increased when contact was made with families by telephone or post to gather family data prior to the offer of the appointment (Mathai \& Markantonakis, 1990). $A$ recent study showed that attendance rates could be increased in a general adult psychiatry clinic by sending patients a reminder within a few days of their appointment (Rusius, 1995).

Asking patients to confirm in advance whether or not they require an appointment has been shown to reduce significantly the non-attendance rate in two studies. Baggaley (1993) showed a reduction in the non-attendance rate from $28 \%$ to $3 \%$ in general psychiatry patients but reported that the proportion of all referrals seen fell from $72 \%$ to $61 \%$, presumably because some patients who would have attended falled to contact the clinic for an appointment. A significant reduction in the non-attendance rate from $50 \%$ to $34.5 \%$ was also found following the introduction of a similar system in an alcohol clinic (Goldbeck, 1993). Again there was a fall from $50 \%$ to $42 \%$ in the overall proportion of total referrals seen.

In this study, an attempt was made to increase the attendance rate for new referrals to a community-based alcohol service by employing a new appointments system similar to those described in these two studies (Baggaley, 1993; Goldbeck, 1993). Clinics were already held in health clinics rather than hospitals and personal letters of invitation were already employed (Hills \& Alexander, 1990).

\section{The study}

This was a retrospective study of new alcohol referrals to a community drug and alcohol service serving Mid Glamorgan, population 544 300. Prior to January 1994 all referred patients were routinely sent an appointment and those who failed to attend were sent a second and sometimes a third appointment. A new system was adopted which involved writing to all referred patients asking them to contact the clinic by telephone to confirm whether or not they required an appointment. Those who failed to contact the clinic within 8 weeks were not sent an appointment and the referrer was advised that the patient had not responded.

The study involved analysis of case records of new referrals for alcohol problems, excluding self-referrals, referrals from probation officers or solicitors, and domiciliary or hospital liaison requests. Demographic characteristics, attendance rates and intervals between referral and appointment dates were collected for all relevant patients. The first three months of the new system were excluded to avoid possible complications arising from the change-over period and any teething problems with the system. To reduce the possible confounding effect of seasonal variation 
in attendance rates, referrals from 1 April 1993 were compared with referrals from 1 April 1994.

\section{Findings}

There were 120 alcohol referrals between 1 April and 31 December 1993, of which 80 met inclusion criteria for the study, and 125 referrals between 1 April and 3 October 1994 of which 90 met inclusion criteria for the study.

There were no significant differences in demographic characteristics between the two groups: the mean age was 39.8 years and the male to female ratio was approximately 2:1. There were similar mean intervals between the date on which the referral was received by the service and the date of the appointment in both groups (44 and 47 days). Those who attended their appointments waited longer for that appointment than those who did not attend under both the old (49 v. 37 days) and new systems (49 v. 42 days).

Under the old system 48 of 80 patients attended their first appointment, a non-attendance rate of $40 \%$. Under the new system 42 of 90 patients did not ring to confirm that they wanted an appointment and one person rang to indicate that they did not wish to be seen. Of the 47 patients who rang to confirm their wish to be seen, 31 attended their first appointment, a nonattendance rate of $33 \%$. The difference failed to reach statistical significance. Under the old system $60 \%$ of all referrals were seen at their first appointment, compared with $34 \%$ under the new system $(P<0.001)$.

\section{Comment}

Our findings are consistent with those of the two previous reports (Baggaley, 1993; Goldbeck, 1993) which suggested that asking patients to make contact with a clinic to confirm that they want an appointment can reduce non-attendance rates in those who respond. This is unsurprising as those with sufficient motivation and organisational ability to reply are, at face value, more likely to attend.

If the data from the current study are pooled with those of Baggaley (1993) and Goldbeck (1993), to make an aggregated total sample size of 608 referrals, then while there is a significant fall in the rate of non-attendance from $42 \%$ to $27 \%(P<0.01)$, this has to be set against a significant overall reduction in the proportion of all referrals assessed, from $48 \%$ to $38 \%$ $(P<0.001)$.

The low rate of contacting the service for appointments may be explained by a number of factors, some of particular relevance to patients with psychiatric and alcohol problems. Denial and lack of insight, the stigma associated with seeking help, fluctuating motivation to address one's problems and low self-esteem may all discourage the patient from contacting the clinic. Limited availability of a confidential telephone line may also have been of significance in the socio-economically deprived population under study.

These new appointment systems clearly produce potential savings in professional's time but at the expense of accessibility to patients who may be those with most need. In the current study, as many as $43 \%$ of those who would have attended under the old system were not seen. A large proportion of patients were thus deprived of the opportunity for assessment and access to treatment.

Alternative options to the system described in this paper merit further investigation to minimise wasted staff time while retaining and maximising accessibility. Clear communication with and education of referrers might help to reduce inappropriate and obviously unwilling referrals. Moving clinics away from psychiatric hospitals may be impractical in some services, but sending personal letters rather than cards (Hillis \& Alexander, 1990) and following up all appointment letters with reminders (Rusius, 1995) could relatively easily be introduced into most administrative systems. Collecting preassessment information by telephone or postal questionnaire might be investigated for adult patients as it has been shown to be successful with families (Mathai \& Markantonakis, 1990). In addition, an alternative to telephone or postal contact might be to book all referred patients in to 15-minute, pre-interview assessments with a nurse. At this meeting personal contact could be established, anxieties allayed, routine data and rating scales completed and laboratory screening tests carried out, so that all these data could be available at the time of the assessment interview proper. With only 15 minutes of staff time wasted per non-attender, and the investment made by the patient in the 15 minutes, we would expect that the second, full assessment interview would invariably be an appointment to be kept.

\section{References}

ADDENBROOKE, W. M. \& RATHOD, N. N. (1990) Relationship between waiting time and retention in treatment amongst substance abusers. Drug and Alcohol Dependence, 26, 255-264.

BAGGALEY, M. (1993) Improving the attendance for new psychiatric out-patient referrals. Psychiatric Bulletin. 17. 347-348.

CotTrell, D., Dearnaley, J., Hill, P., et al (1988) Factors influencing non-attendance at child psychiatry outpatient appointments. British Journal of Psychiatry. 152. 201-204. 
DAVISON, P. S. (1992) Hand-written appointment letters. Psychiatric Bulletin, 16, 179.

GOLDBECK. R. (1993) Can a card mailing system help to reduce the waiting list at an alcohol problems clinic? Psychiatric Bulletin, 17, 597-598.

GouLD, M. S., KAPLAN, D. \& SHAEFFER, D. (1985) The characteristics of dropouts from a child psychiatric clinic. Journal of the American Academy of Child Psychiatry, 24, 316-328.

HILUS, G. \& ALEXANDER, D. A. (1990) Rejection of psychiatric treatment. Psychiatric Bulletin, 14, 149-150.

MATHA, J. \& MARKANTONAKIS, A. (1990) Improving initial attendance to a child and family psychiatric clinic. Psychiatric Bulletin, 14, 151-152.

PLUNKETT. J. W. (1984) Parents' treatment expectations and attrition from a child psychiatric service. Clinical Psychology, 40, 372-377.
RUSIUS, C. W. (1995) Improving out-patient attendance using postal appointment reminders. Psychiatric Bulletin, 19, 291-292.

SKUSE, D. (1975) Attitudes to the psychiatric out-patient clinic. British Medical Journal, 3. 469-471.

*Imad M. Ali, Senior Registrar, Gwent Community Health NHS Trust, Talygarn County Hospital, Griffithstown. Pontypool, Gwent; and Andrew J. McBride, Consultant Psychiatrist, Community Addiction Unit, Cardiff Community Healthcare NHS Trust, Whitchurch Hospital, Whitchurch, Cardiff

*Correspondence

\section{College Seminars Series

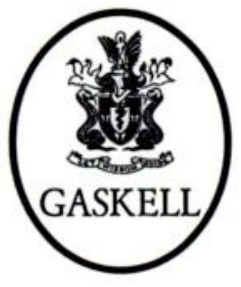 \\ Seminars in Alcohol and Drug Misuse \\ Edited by Jonathan Chick and Roch Cantwell}

Psychiatric complications of alcohol and drug misuse must be recognised and treated confidently. This book provides information to meet the needs of practising psychiatrists and will be useful to physicians, psychologists and social workers. A clear review of the aetiology, epidemiology, treatment and prevention of dependence on and misuse of alcohol and illicit and prescribed drugs is presented. With a balance of theory, recent research and practical clinical guidelines, the book covers specific and common problems in mental health as well as in general medicine. El3.50, 1994, 256pp. ISBN $090244 \mid 702$

Available from good bookshops and from the Publications Department, Royal College of Psychiatrists, 17 Belgrave Square, London SWIX 8PG. Credit card orders can taken over the telephone $(+44(0) I 7 \mid-235235 I$, extension I46). 